\title{
Design and Simulation of UHF RFID Tag Antennas and Performance Evaluation in Presence of a Metallic Surface
}

\author{
Tashi $^{1,2}$, Mohammad S. Hasan ${ }^{2}$, and Hongnian $\mathrm{Yu}^{2}$ \\ ${ }^{1}$ Department of Electrical Engineering, College of Science and Technology, Rinchending, Phuentsholing, Bhutan \\ ${ }^{2}$ Faculty of Computing, Engineering and Technology, Staffordshire University, Stafford, Staffordshire, ST18 0AD, UK \\ ${ }^{1}$ tashi@cst.edu.bt, ${ }^{2}\{$ m.s.hasan, h.yu\}@staffs.ac.uk
}

\begin{abstract}
Passive UHF RFID system have drawn considerable attention because it can provide a long reading range, high data rate, and small antenna size. However, a passive UHF RFID system has challenges in tagging a metallic object due to effects of conducting materials on the tag antenna performance. This study presents investigation and analysis of effects of a metallic surface on the performance of commonly used label-type dipole tag antenna. The performance is evaluated based on the return loss and change in resonance frequency of the tag antenna. To conduct the performance analysis, a folded dipole and a meandered dipole antenna are proposed for a passive tag. The proposed antennas are designed on low profile antenna which can operate at $866 \mathrm{MHz}$ in the UK and Europe UHF RFID frequency band. The design also takes into account of impedance matching, feeding techniques, size-reduction, and bandwidth of the passive UHF tag antenna. Finally, simulation result shows that when the tag antenna is mounted closer to a metallic surface, the return loss of tag antenna is significantly increased and the resonance frequency is also shifted from the desired frequency.
\end{abstract}

Index Terms - Effect of metal, folded dipole antenna, meandered dipole antenna, tag antenna performance.

\section{INTRODUCTION}

$\mathrm{T}$ he rapid increase on requirements of automatic identification in various areas such as item-level tracking, access control, electronic toll collection, vehicle security [1] etc. accelerates the demand for the radio frequency identification (RFID).

RFID is a pervasive computing technology [2]. It consists of a reader, tag, and application computer. A basic configuration of passive RFID system is shown in Fig. 1. Based on the method of powering, RFID system is classified as a passive (without battery), active (with battery), and semiactive. RFID system is also classified as the near-field and farfield system based on the method of coupling between the reader and tag. The near-field works on the electromagnetic induction and it operate at low frequency (LF) around 125-134 $\mathrm{kHz}$ or high frequency (HF) at $13.8 \mathrm{MHz}$ [3]. On the other hand, the far-field works on the backscattering propagation and it operate at ultra-high frequency (UHF) around 860-960 $\mathrm{MHz}$ or microwave frequency (MWF) at $2.5 \mathrm{GHz}$ [3].

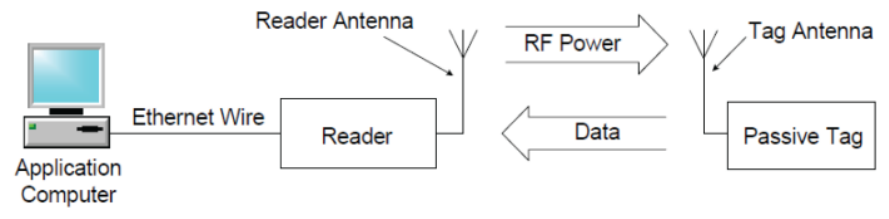

Fig. 1. A basic configuration of passive RFID system.

In recent years, passive UHF RFID system is getting considerable attention because it can provide a long reading range, high data rate, and small antenna size. Many applications require tag antenna to be of low profile and easy to be mounted or embedded on any objects. A low-cost labeltype dipole antenna printed on a very thin film is commonly used as the tag antennas in passive UHF RFID applications [4, 5]. However, when mounted on a metallic object, the antenna parameters such as radiation pattern, bandwidth, gain, input impedance etc. change and the overall efficiency seriously degrades due to the change of the reactance of the antenna impedance $[6,7]$. In the worst case, the tag may be not be detected by the reader within its normal reading range.

The tag antenna problems for RFID system is first reported by Foster and Burberry [8]. Only limited work on the effect of metal near the antenna has been published. The effect of metallic reflector on the folded dipole antenna is simulated by Raumonen et al [9] and it has been reported that there is decrease in radiation pattern when the antenna is taken closer to the metallic reflector. Dobkin and Weignand [6] have shown the experimental set-up to measure the reading distance of a tag near metal plate and water filled container. They have reported that there is a decrease in reading distance when the tag is close to the metal plate. They also reported changes in the tag antenna impedance when the tag is taken near to a metallic plate. In [7], the power and backscatter communication radio link budgets that allow the tag designer to quantify the effects of tag material attachment is studied. And they have reported a decrease in tag antenna gain when placed near the metallic surface. The effects on the antenna parameters when placed on paper and plastic is studied by Radi et al [10] and it has been reported that there are shift in resonance frequency when the antenna substrate are changed. Mittra and Hoenschel [11] have designed a tag antenna that employs Electromagnetic Bandgap (EBG) surfaces substrate to have less sensitivity to the environment. However, their tag antenna parameters like radiation pattern and impedance characteristics change little when placed on metal or glass. The effect of the substrate, metal-line and surface material on the performance of RFID tag antenna is investigated in [12]. This paper has reported that the readability of the tag drastically decreases when the tag is attached to a high permittivity and high loss target object. Paper [13] has studied performance of the RFID tag antenna based on the antenna trace materials like copper, aluminium and sliver. It has been 
observed that copper deposit and silver inks are competitive materials compared to aluminium when using $1 \mathrm{~mm}$ antenna trace width. However, deposited copper has superior conductivity, and can work with much less deposited material than silver and with narrow line widths.

In this paper, the effect of a metallic surface on the return loss and change in resonance frequency of label-type dipole antenna is studied and analysed. Two variants of dipole antenna; folded dipole and meandered dipole antenna are proposed to study the effect of metallic surface. First the proposed tag antennas are designed and analysed in free space. Then, the effects of a metallic surface on the proposed antennas are analysed. The rest of the paper is organised as follows. Section II discusses the proposed antenna designs. Section III presents simulation results of the proposed antennas. Section IV addresses the performance of proposed tag antennas near or on metallic surface. Finally, section V draws some conclusions.

\section{THE PROPOSED ANTENNA DESIGN}

The proposed folded dipole and meandered dipole antenna design in presented in the following sections.

\section{A. Folded Dipole Antenna}

The geometry of the proposed folded dipole antenna is shown in Fig. 2 and is considered to use in a smart label for cardboard box tagging in warehouse environment. It should be tuneable in range of 865 to $868 \mathrm{MHz}$ to operate in the UK and Europe UHF RFID frequency band. Therefore, the operating frequency of the proposed tag antenna is selected as $866 \mathrm{MHz}$. It is printed on a thin polyimide substrate with a dielectric constant $\left(\varepsilon_{r}\right)$ of 3.5 and loss tangent $(\delta)$ of 0.003 . The thickness $\left(t_{s}\right)$ of the substrate is $0.05 \mathrm{~mm}$. This substrate is very cheap and it can be easily mounted to the cardboard box. The top antenna trace is made of copper with thickness $\left(t_{a}\right)$ of 0.018 $\mathrm{mm}$. The Alien Higgs-3 [14] is selected as the tag chip, which exhibits an impedance of $Z_{C}=(31-\mathrm{j} 212) \Omega$ at $866 \mathrm{MHz}$ resonance frequency. In order to deliver maximum power from the tag antenna to chip, the input impedance of the proposed tag antenna should be $Z_{A}=(31+\mathrm{j} 212) \Omega$ at the resonance frequency. The proposed folded dipole antenna has two elements: main radiating element and parasitic element. The key antenna parameters are: antenna length $L$, antenna breath $B$, antenna trace width $w$, folded arm length $a$, parallel distance $d$ between the main element and the parasitic element, and the separation gap between folded arm and parasitic element $s$.

\section{B. Meandered Dipole Antenna}

The geometry of the proposed meandered dipole antenna is shown in Fig. 3. The operating frequency, chip impedance, substrate properties and application of the proposed meandered dipole antenna is considered the same as the proposed folded dipole antenna discussed in section II.A. The T-match feeding technique is used to feed the proposed antenna to match the antenna impedance to the chip impedance. The proposed meandered dipole antenna has several key parameters: length of antenna $L$, meander step height $h$, meander step width $w$, conductor spacing $s$, length of last meandered conductor $B$, and the separation distance between the feed element and main radiating element $d$.

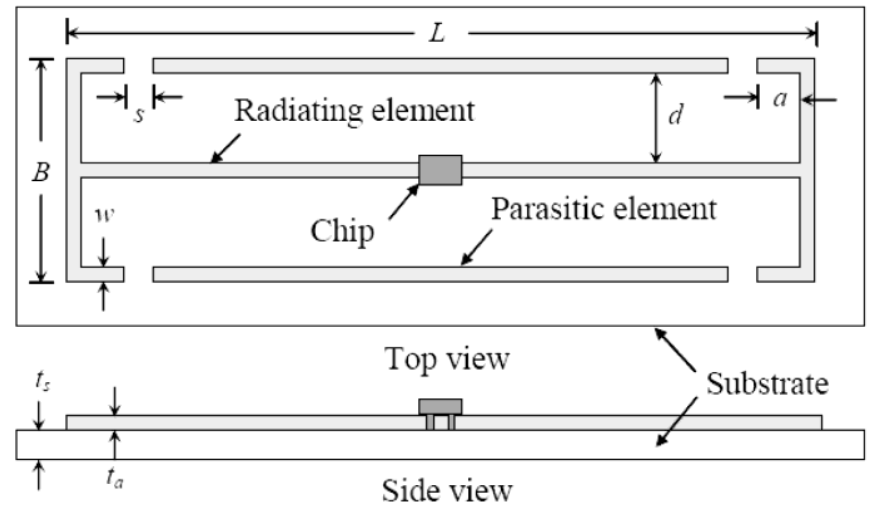

Fig. 2. Geometry of the proposed folded dipole antenna.

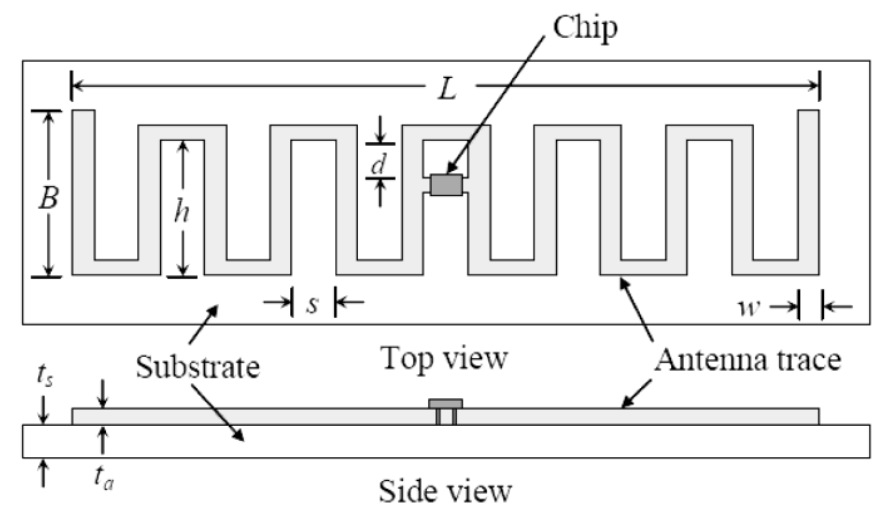

Fig. 3. Geometry of the proposed meandered dipole antenna.

\section{SIMULATION RESULTS}

The proposed tag antennas are simulated in the Sonnet Lite electromagnetic (EM) simulator [15] which simulates based on the method of moments (MoM).

\section{A. Folded Dipole Antenna}

A numerous parameter sweep are performed in the simulation and each result is examined. The final optimised parameters of the proposed folded dipole antenna are given in Table I. Fig. 4 shows the input resistance against UHF frequency of the proposed folded dipole antenna with various separation gaps, $s$. Fig. 5 shows the simulated input reactance against UHF frequency of the proposed folded dipole antenna with various separation gap, $s$. It is clear that input resistance and reactance can be increased or decreased by varying $s$. The simulation result shows that when $s=5 \mathrm{~mm}$, the antenna input impedance is complex conjugately matched to the chip input impedance.

Fig. 6 presents the return loss of the proposed folded dipole antenna at $866 \mathrm{MHz}$ resonance frequency. The minimum value of the simulated return loss $\left(\mathrm{S}_{11}\right)$ at resonance frequency is $-21.59 \mathrm{~dB}$. At half-power return loss $(<-3 \mathrm{~dB})$, the proposed folded dipole antenna covers entire UHF RFID frequency range (i.e. $860 \mathrm{MHz}$ to $960 \mathrm{MHz}$ ) and the proposed antenna 
can be used in the world wide UHF RFID system. The simulated $<-10 \mathrm{~dB}$ bandwidth of the proposed antenna is 67.5 $\mathrm{MHz}(7.79 \%)$, from $836 \mathrm{MHz}$ to $903.5 \mathrm{MHz}$ which can easily operate in the UK and Europe UHF RFID system. Both $-3 \mathrm{~dB}$ and $-10 \mathrm{~dB}$ bandwidth meet the requirement $(500 \mathrm{kHz})$ of the ISO/IEC 18000-7 standard [16].

\section{TABLE I}

PARAMETERS OF THE PROPOSED FOLDED DIPOLE ANTENNA (DIMENSION IN MM)

\begin{tabular}{|c|c|c|c|c|c|c|}
\hline Parameters & $L$ & $B$ & $s$ & $w$ & $a$ & $d$ \\
\hline Values & 108 & 28 & 4 & 2 & 6 & 12 \\
\hline
\end{tabular}

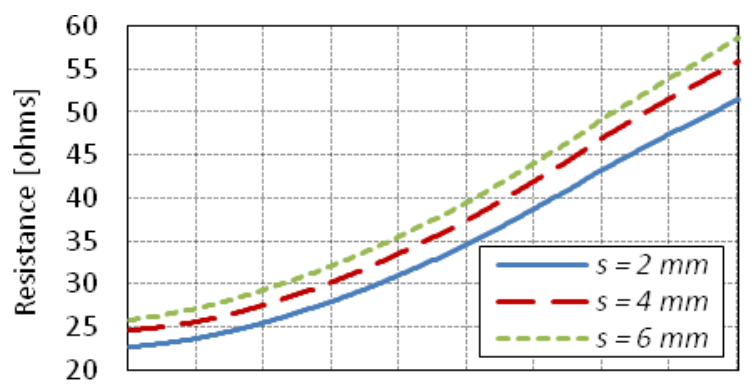

$\begin{array}{llllllllll}800 & 820 & 840 & 860 & 880 & 900 & 920 & 940 & 960 & 980\end{array}$ Frequency $[\mathrm{MHz}]$

Fig. 4. Simulated input resistance against UHF frequency of the proposed folded dipole antenna with various separation gaps, $s$.

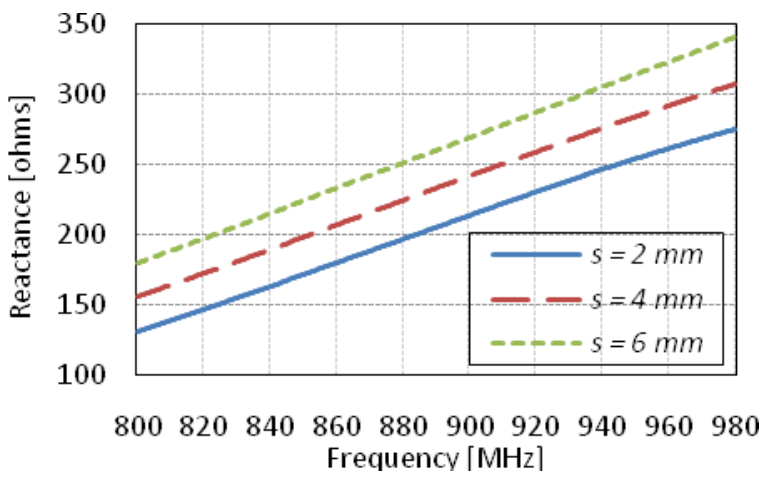

Fig. 5. Simulated input reactance against UHF frequency of the proposed folded dipole antenna with various separation gaps, $s$.

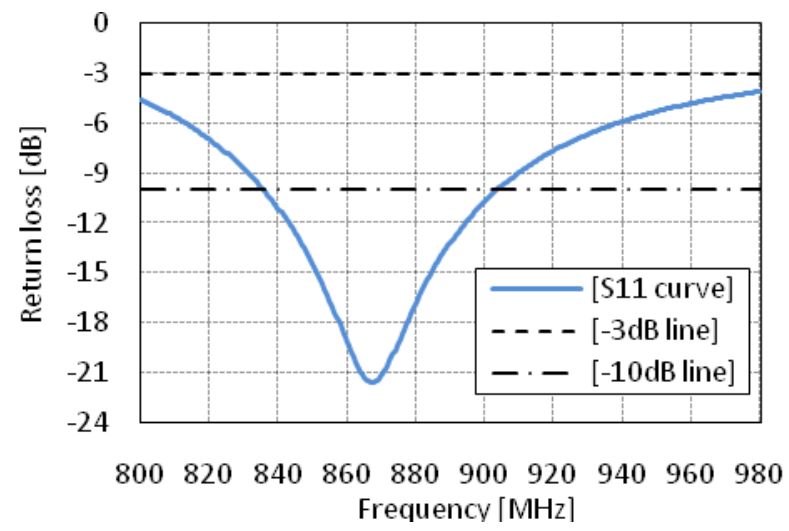

Fig. 6. Simulated return loss of the proposed folded dipole antenna at 866 $\mathrm{MHz}$ resonance frequency.

\section{B. Meandered Dipole Antenna}

Table II presents the final optimised parameters of the proposed meandered dipole antenna. Fig. 7 shows the simulated input resistance against UHF frequency of the proposed meandered dipole antenna with various separation distances, $d$. Fig. 8 shows the simulated input reactance against UHF frequency of the proposed meandered dipole antenna with various separation distances, $d$. It is clear that input resistance and reactance can be increased or decreased by varying $d$. The simulation result shows that when $d=5$ $\mathrm{mm}$, the antenna input impedance is complex conjugately matched to the chip input impedance.

Fig. 9 presents the return loss of the proposed meandered dipole antenna at $866 \mathrm{MHz}$ resonance frequency. The minimum value of the simulated return loss $\left(\mathrm{S}_{11}\right)$ at the resonance frequency is $-22.76 \mathrm{~dB}$. The half-power bandwidth (return loss < $-3 \mathrm{~dB}$ ) is $77 \mathrm{MHz}(8.89 \%)$, from $829.5 \mathrm{MHz}$ to 906.5 MHz. The simulated $<-10 \mathrm{~dB}$ bandwidth of the proposed antenna is $25 \mathrm{MHz}$ (2.89\%), from $855 \mathrm{MHz}$ to 880 MHz. Both at $-3 \mathrm{~dB}$ and $-10 \mathrm{~dB}$ return loss, the proposed tag antenna can be used in the UK and Europe UHF RFID system. Also $-3 \mathrm{~dB}$ and $-10 \mathrm{~dB}$ bandwidth of the proposed antenna meet the requirement of $(500 \mathrm{KHz})$ of the ISO/IEC 18000-7 standard [16].

TABLE II

PARAMETERS OF THE PROPOSED MEANDERED DIPOLE ANTENNA (DIMENSION IN MM)

\begin{tabular}{|c|c|c|c|c|c|c|}
\hline Parameters & $L$ & $B$ & $h$ & $w$ & $s$ & $d$ \\
\hline Values & 68 & 22 & 18 & 2 & 4 & 5 \\
\hline
\end{tabular}

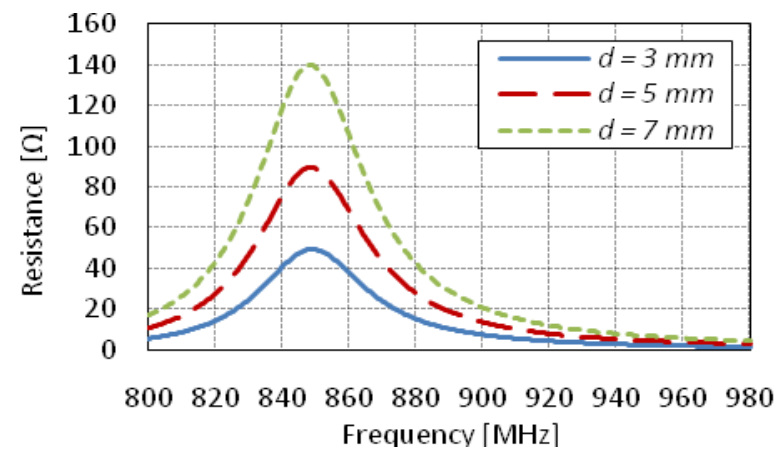

Fig. 7. Simulated input resistance against UHF frequency of the proposed meandered dipole antenna with various separation distances, $d$.

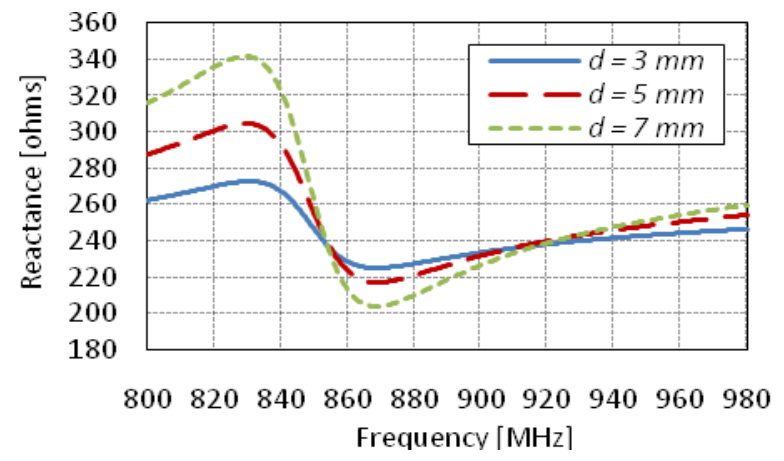

Fig. 8. Simulated input reactance against UHF frequency of the proposed meandered dipole antenna with various separation distances, $d$. 


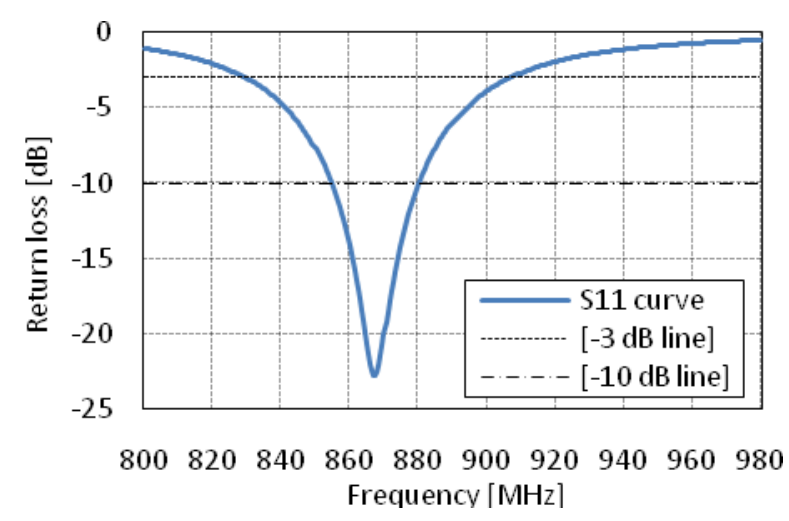

Fig. 9. Simulated return loss of the proposed meandered dipole antenna at 866 $\mathrm{MHz}$ resonance frequency.

\section{Comparison of the Proposed Folded and Meandered Dipole Antenna}

Fig. 10 shows the comparison for the area of the proposed folded dipole and meandered dipole antenna. By using the meandered dipole antenna, an area reduction of $50.53 \%$ can be achieved. Therefore, meandered dipole antenna would offer a compact and robust passive tag which is demanded by most of the RFID applications. However, the proposed meandered dipole antenna suffers from a narrower bandwidth characteristics than the folded dipole antenna as shown in Fig. 11. Therefore, a trade-off or compromise among the area, bandwidth, and performance parameters is needed based on the type of tag antenna and the RFID applications. For example, if the size of tag requirement for RFID is a crucial factor, then the meandered dipole antenna can be used.

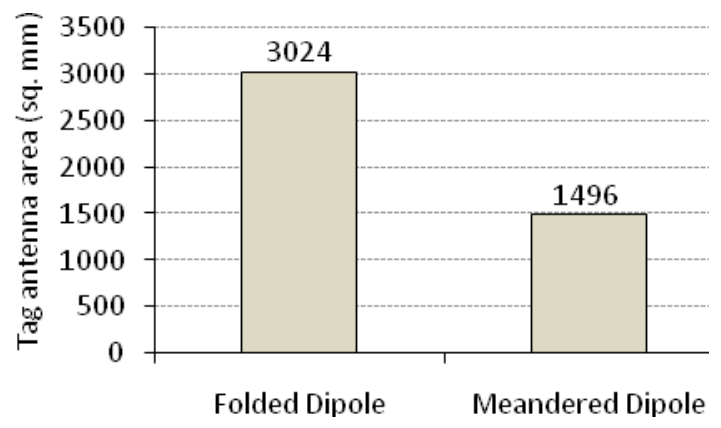

Fig. 10. The area comparison of the proposed tag antennas.

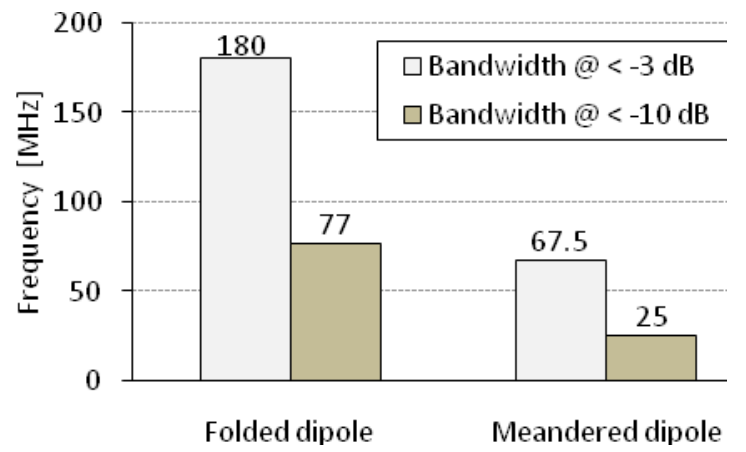

Fig. 11. The bandwidth comparison for the proposed tag antennas at $<-3 \mathrm{~dB}$ and $<-10 \mathrm{~dB}$ return loss.

\section{PeRformance Of the TAG ANTENNA WHEN PLACED ON A METALLIC SURFACE}

The simulation environment to investigate the performance of tag antenna when mounted on a metallic surface is set-up in the Sonnet lite EM simulator. The effect of a metallic surface on the tag antenna is evaluated by the return loss and resonance frequency of the tag antenna. Fig. 12 shows how a tag antenna is mounted on a metallic surface in the EM simulator to evaluate the effect of a metallic surface. The tag antenna is placed above the metallic surface keeping air as the medium between them. The separation gap between the tag antenna and metal is $d$. The infinite ground plane of the EM simulator is used as a metallic surface which is $0.2 \mathrm{~mm}$ thick. The type of the metallic surface for this analysis is considered as copper. The metallic surface is taken closer to the tag antenna by step $d=10 \mathrm{~mm}$ in each step. At every step, the return loss value, bandwidth and resonance frequency are examined.

After performing a numerous number of simulations, it is observed that when the tag antenna is placed above the metallic surface more than the quarter wavelength of the antenna's resonance frequency, there is not much by effect metallic surface. Therefore, the performance evaluation emphasises when the distance between the tag antenna and a metallic surface is less than quarter of the wavelength.

Fig. 13 and Fig. 14 show the simulation results of the proposed folded dipole and meandered dipole antenna, respectively for various separation gaps $d$. In both cases, when the tag is taken closer to a metallic surface by step $d=10 \mathrm{~mm}$, there is a significant increase in the return loss and change in the resonance frequency from the desired frequency. The return loss and resonance frequency in the free space for a folded and meandered dipole antenna are shown in Fig. 6 and Fig. 9, respectively. When $d$ is less than $40 \mathrm{~mm}$, the return losses of the proposed antennas are greater than $-3 \mathrm{~dB}$ and the tag antenna may not work at all since it is short circuited by the metallic surface and suffers from a high return loss. The increase in the return loss is due to the change in the antenna impedance according to (1) which is taken from [17],

return loss $=-20 \log _{10}\left(\frac{Z_{A}+Z_{C}}{Z_{A}-Z_{C}}\right) d B$

where, $Z_{A}$ is antenna impedance and $Z_{C}$ is chip impedance.

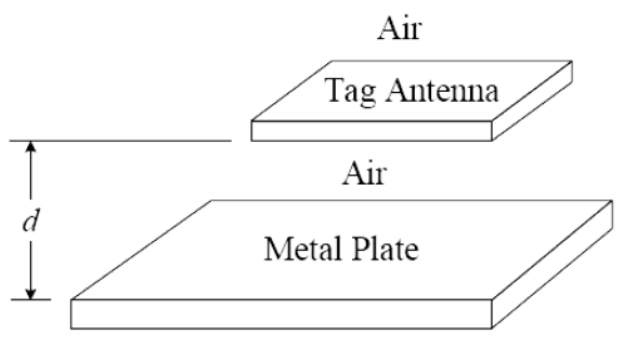

Fig. 12. The isometric view of the simulation model set-up to investigate the performance of a tag antenna on metallic surface. 


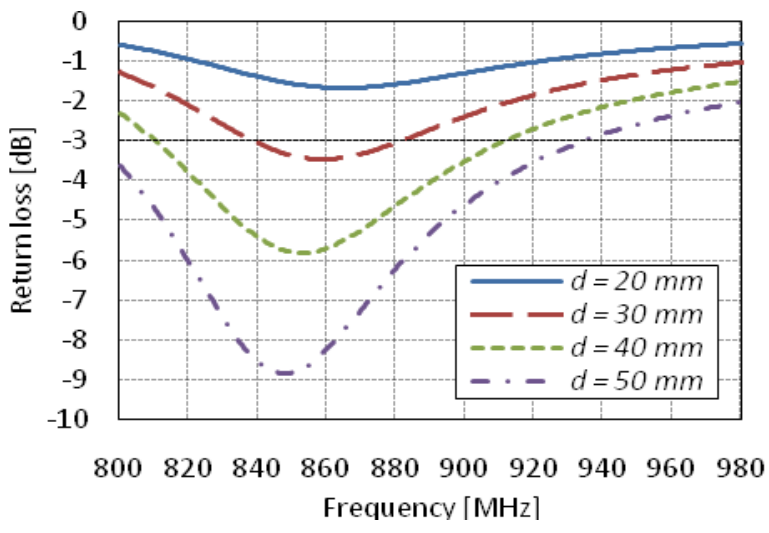

Fig. 13. The return loss of the proposed folded dipole antenna for various separation gaps $d$ between the antenna and metallic surface.

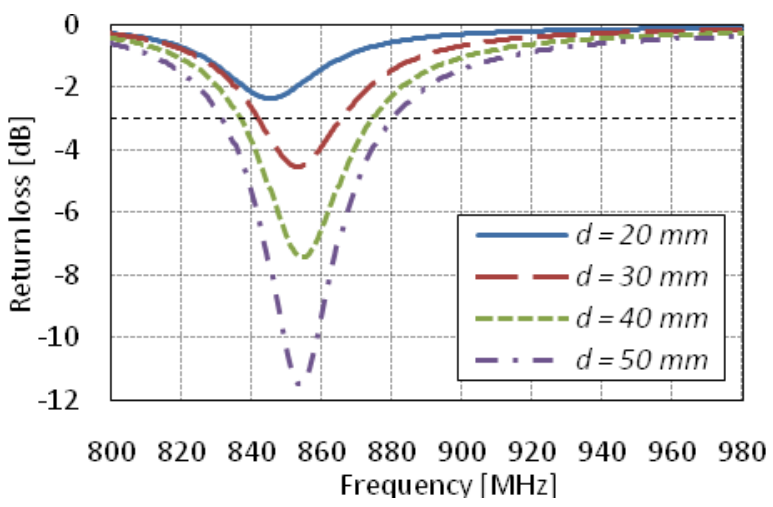

Fig. 14. The return loss of the proposed meandered dipole antenna for various separation gaps, $d$ between the antenna and metallic surface.

\section{CONCLUSION}

In this paper, a folded dipole and a meandered dipole antenna are proposed on very low profile for a passive UHF RFID tag. The simulation results show that the meandered dipole antenna structure offers a compact and small tag size. However, it suffers from the narrow bandwidth. The performance of the proposed tag antennas on a metallic surface is studied and analysed. The study shows that the tag antenna is short circuited by a metallic surface when the tag antenna is taken closer to a metallic surface. Thus, return loss increases due to the change in the tag antenna impedance. It results in an impedance mismatch between the tag antenna and chip. Therefore, power supply to the chip is reduced and the tag may not be detected by the reader within the normal reading range. So the possible solution to improve the performance may be by designing an antenna which uses metallic surface as the ground plane such as Micro-strip patch antenna and planar inverted-F antenna (PIFA). The research team has already done some investigations on designing a micro-strip patch antenna prototype for passive UHF RFID tag which will be published in a future paper.

\section{ACKNOWLEDGMENT}

This work was supported by the eLINK (east-west Link for Innovation, Networking and Knowledge Exchange) project.

\section{REFERENCES}

[1] K. Finkenzeller, RFID Handbook Fundamentals and Applications in Contactless Smart Cards and Identification. Chichester: John Wiley and Sons Ltd, 2003.

[2] R. Want, "An introduction to RFID technology," IEEE Pervasive Computing, vol. 5, no.1, pp. 25-33, 2006.

[3] G. Backhouse. (2006). RFID: Frequency, standards, adoption and innovation. JISC Technology and Standards Watch. Available: http://www.jisc.ac.uk/media/documents/techwatch/tsw0602.pdf [Accessed: 07 Jan. 2010].

[4] K. V. S. Rao, et al., "Antenna design for UHF RFID tags: a review and a practical application," IEEE Transactions on Antennas and Propagation, vol. 53, no.12, pp. 3870-3876, 2005.

[5] C. Cho, et al., "Broadband RFID tag antenna with quasi-isotropic radiation pattern," Electronics Letters, vol. 41, no.20, pp. 1091-1092, 2005.

[6] D. M. Dobkin and S. M. Weigand, "Environmental effects on RFID tag antennas," in Microwave Symposium Digest, 2005 IEEE MTT-S International, 2005, pp. 135-138.

[7] J. D. Griffin, et al., "RF Tag Antenna Performance on Various Materials Using Radio Link Budgets," Antennas and Wireless Propagation Letters, IEEE, vol. 5, no.1, pp. 247-250, 2006.

[8] P. R. Foster and R. A. Burberry, "Antenna problems in RFID systems," in IEE Colloquium on RFID Technology 1999, pp. 3/1-3/5.

[9] P. Raumonen, et al., "Folded dipole antenna near metal plate," in Antennas and Propagation Society International Symposium, 2003. IEEE, 2003, pp. 848-851 vol.1.

[10] A. H. Rida, et al., "Design, Development and Integration of Novel Antennas for Miniaturized UHF RFID Tags," Antennas and Propagation, IEEE Transactions on, vol. 57, no.11, pp. 3450-3457, 2009.

[11] R. Mittra and A. Hoenschel, "Some Novel Designs for RFID Antennas and their Performance Enhancement with Metamaterials," in Anticounterfeiting, Security, Identification, 2007 IEEE International Workshop on, 2007, pp. 1-3.

[12] C. Chihyun, et al., "Effect of the substrate, metal-line and surface material on the performance of RFID tag antenna," in Antennas and Propagation Society International Symposium, 2007 IEEE, 2007, pp. 1761-1764.

[13] A. Syed, et al., "Effects of Antenna Material on the Performance of UHF RFID Tags," in RFID, 2007. IEEE International Conference on, 2007, pp. 57-62.

[14] Alien. (2010), RFID ICs. Available: http://www.alientechnology.com/docs/products/DS_H3.pdf [Accessed: 25 Dec. 2010]

[15] C. Blair and J. C. Rautio, "RFID design using EM analysis," in Applications and Technology Conference (LISAT), 2010 Long Island Systems, 2010, pp. 1-6.

[16] L. Xu, et al., "UHF RFID tag antenna with broadband characteristic," Electronics Letters, vol. 44, no.2, pp. 79-80, 2008.

[17] T. S. Bird, "Definition and Misuse of Return Loss [Report of the Transactions Editor-in-Chief]," Antennas and Propagation Magazine, IEEE, vol. 51, no.2, pp. 166-167, 2009. 